\title{
Trichoderma atroviride: an isolate from forest environment with secondary metabolites with high antimicrobial potential
}

\author{
Ján Víglaš, Petra Olejníková \\ Institute of Biochemistry and Microbiology, Slovak University of Technology in Bratislava, \\ Radlinského 9, 81237 Bratislava, Slovakia \\ jan.viglas@stuba.sk
}

\begin{abstract}
This work was focused on the characterization of novel isolate of Trichoderma atroviride O1, found in the forest around the village of Zázrivá (the Northern Slovakia, region Orava). The isolate was identified by sequencing its internal transcribed spacer (ITS) region of rDNA. T. atroviride O1 stimulated the development of lateral roots of model plant Lepidium sativum. Simultaneously, the isolate has proved its high mycoparasitic potential as it displayed the ability to attack colonies of phytopathogenic fungi (Alternaria alternata, Fusarium culmorum, Botrytis cinerea). This isolate produced secondary metabolites, which were isolated and tested for the antimicrobial activity against gram-positive bacteria Staphylococcus epidermidis and Staphylococcus aureus. The growth of these bacteria was suppressed to $10 \%$ and $40 \%$, respectively. The suppression of the growth of two Candida species was also strong (10\% growth). However, growth parameters of three phytopathogenic fungi (Alternaria alternata, Botrytis cinerea and Fusarium culmorum) were less affected (75\% growth in comparison with the control). Attempts were made to characterize secondary metabolites isolated from T. atroviride $\mathrm{O}$. Known peptaibols, 20-21 amino acid long, but also shorter peptides, were detected by MALDI-TOF mass spectrometry. Thus, this study demonstrates the plant growth promotion, strong mycoparasitic potential and antimicrobial activity of the isolate $T$. atroviride $\mathrm{O} 1$, which could be in part ascribed to the production of secondary metabolites. This isolate does have a potential in the biocontrol in eco-farming. Further study, particularly, the identification of produced secondary metabolites, is needed.
\end{abstract}

Keywords: antimicrobial activity, mycoparasitism, plant-growth promotion, secondary metabolites, Trichoderma

Abbreviations: CCM - Czech Collection of Microorganisms, CWDE - cell wall degrading enzymes, DMSO - dimethylsulfoxide, GKCH - Sabouraud Chloramphenicol agar, ITS - internal transcribed spacer, MALDI-TOF - Matrix Associated Laser Desorption Ionisation with Time of Flight detector, MHA - Mueller Hinton Agar, NRPs - non-ribosomal peptides, PDA - Potato Dextrose Agar, SMs - secondary metabolites, TLC - Thin Layer Chromatography, 6-PP - 6-pentyl- $\alpha$-pyrone

\section{Introduction}

Trichoderma (teleomorph Hypocrea) is a genus of typical soil filamentous fungi. It obtains nutrients through the three different lifestyles: saprophytic, symbiotic and parasitic one. Trichoderma reesei is a typical saprophyte. It colonizes dead woody and herbaceous material and produces cellulolytic and hemicellulolytic enzymes to digest substrate. Interaction with plants is an example of a symbiotic lifestyle. Plant root exudates are a valuable source of nutrients, while Trichoderma sp. promotes plant growth and provides defense against biotic and abiotic stress. Other enzymatic apparatus is involved in parasitic interactions with plant pathogenic fungi, so-called cell wall degrading enzymes that are chitinases, glucanases, and proteases (Druzhinina et al., 2011; Harman et al., 2004). These findings suggest Trichoderma sp. can combat phytopatho- genic fungi. It is just plant-growth promotion and mycoparasitism that mark the potential of Trichoderma sp. in the area of biocontrol agents. In fact, European Commission has approved five species as biopesticides: Trichoderma atroviride (strain IMI 206040, T11, AGR2, I-1237, SC1); Trichoderma asperellum (strain ICC012, T25, TV1 and T34); Trichoderma gamsii (strain ICC080); Trichoderma harzianum (strain T-22 and ITEM 908); Trichoderma polysporum (IMI 206039) [http://ec.europa.eu/food/plant/ pesticides/eu-pesticides-database].

However, Trichoderma-plant and Trichoderma-phytopathogenic fungus interactions are much more complex, mainly because they involve not only the direct effect of hydrolytic enzymes, but also the role of secondary metabolites (SMs) (Vinale et al., 2008b; Liu et al., 2016; Alwhibi et al., 2017; Antasova et al., 2013). SMs are numerous heterogeneous groups of compounds, which are not essential for survival but 
are supposed to have functions in signalling and defense (Mukherjee et al., 2013). SMs of filamentous fungi are divided into four groups, according to the origin of synthesis and structure: non-ribosomal peptides (NRPs), polyketides, terpenes and pyrones (Zeilinger et al., 2016).

By interactions with other organisms, compounds of each group are involved, to various extent. Peptaibiotics, specific NRPs consisting of both proteinogenic, as well as non-proteinogenic amino acids (typical is $\alpha$-aminoisobutyric acid $=$ AIB) are intensively studied (Nawrocka and Małolepsza, 2013; Druzhinina et al., 2011; Neuhof et al., 2007). The subfamily of peptaibols, 18-21 amino acids long with C-terminal alcohol, was described to have its function in signalling by activation of induced systemic resistance of plants (Mukherjee et al., 2013; Nawrocka and Małolepsza, 2013; Druzhinina et al., 2011). Peptaibiotics were also described as inhibitors of $\beta$-glucan synthase (an enzyme involved in cell wall synthesis) of plant pathogenic fungi (Vinale et al., 2008a). Alamethicins and paracelsins are particular peptaibols, known to be produced by Trichoderma sp. (Reino et al., 2008), which are involved in previously mentioned processes. One of the best known SMs produced by Trichoderma sp. is 6-pentyl- $\alpha$-pyrone (6-PP) (Collins a Halim, 1972), described as the auxin-like compound in terms of its effect on plant growth, especially enhancement of root and shoot development (Vinale et al., 2008a). Its production was also observed by a mycoparasitic attack on the colony of phytopathogen (Antasova et al., 2013). This volatile organic compound with the aroma of coconut is produced by $T$. atroviride (Druzhinina et al., 2011). Another group of SMs, polyketides, are also known as molecules that have the role in signalling, such as chrysophanol, member of a subgroup of anthraquinonons (Liu et al., 2016). They are synthesized by polyketide synthases; one of them, encoded by the gene $p k s 4$ is responsible for green pigmentation of conidia (Antasova et al., 2013). Out of the last group, terpenes (based on the structural unit of isoprenyl pyrophosphate), the production of trichothecenes is significant. Trichoderma brevicompactum and Trichoderma arundinaceum, which produce trichothecenes can inhibit the growth of Fusarium sp. (dangerous plant pathogen), while Trichoderma gamsii, which has genes for trichothecene production but their own trichothecenes were not detected, appears to tolerate trichothecenes of Fusarium sp. (Tijerino et al., 2011). To sum up, SMs of Trichoderma sp. are studied because of their potential to provide new antimicrobial compounds.

This paper provides a characterization of the isolate of $T$. atroviride $\mathrm{O} 1$, obtained from the forest environment. The paper shows the potential of the obtained isolate in interaction with the plant by promoting its growth; in mycoparasitic activity on phytopathogenic fungi and production of secondary metabolites with antimicrobial activity. Using MALDI-TOF mass spectroscopy, detection of peptaibiotics was performed.

\section{Materials and methods}

\section{Microbial strains and culture conditions}

Isolate Trichoderma atroviride $\mathrm{O} 1$ as well as Trichoderma viride CCM (Czech Collection of Microorganisms): F534, phytopathogenic filamentous fungi: Alternaria alternata CCM: F128, Fusarium culmorum CCM: F21, Botrytis cinerea CGM: F16 were maintained on Potato Dextrose Agar (PDA) (Biolife, Milan, Italy) slants at $4{ }^{\circ} \mathrm{C}$ and sub-cultured on fresh growth medium every two weeks. Fungi were cultivated at the room temperature. Yeast: Candida albicans SC 5341, Candida parapsilosis ATCG 22019 were stored in glycerol $(30 \%)$ at $-80{ }^{\circ} \mathrm{C}$ and three days before experiments were inoculated on GKCH = Sabouraud Chloramphenicol agar (Biorad, Marnes-La-Coquette, France) and cultivated at $37^{\circ} \mathrm{C}$. Model bacteria: Staphylococcus aureus CCM 3953, Staphylococcus epidermidis CGM 4505 and Escherichia coli CCM 3988 were stored in glycerol at $-80{ }^{\circ} \mathrm{C}$ and two days before experiment inoculated on MHA = Mueller Hinton Agar (Biolife, Milan, Italy), and cultivated at $37^{\circ} \mathrm{C}$.

\section{Isolation of Trichoderma sp. \\ from the wood material obtained from the forest in Northern Slovakia - Orava}

The piece of wood with assumed Trichoderma sp. was transferred to the laboratory, conidia (Fig. 1) were stamped at PDA growth medium (Biolife, Milan, Italy). After one week of cultivation at room temperature, part of mycelium was transferred on fresh PDA. Next, we repeated the process until we obtained pure culture (Fig. 1).

\section{DNA isolation and PCR amplification}

The isolate was grown on PDA medium, covered with cellophane, at $25^{\circ} \mathrm{C}$, in the dark for two days. Mycelium was harvested and homogenized in liquid nitrogen. For isolation of genomic DNA, the DNA Fast Isolation Kit (Spin-column) was used (Ecoli s.r.o., Bratislava, Slovakia).

The internal transcribed spacer (ITS) region was amplified using a set of primers ITS1 (forward primer): 5- CTT GGT CAT TTA GAG GAA GTA A - 3' and ITS4 (reverse primer): $5^{\prime}-$ TCC TCG GGT TAT TGA TAT GC - 3' (E.coli s.r.o., Bratislava, Slovakia) and Hot-start DNA poly- 
merase (Biotechrabbit GmbH, Germany). The initial denaturation was held at $94{ }^{\circ} \mathrm{C}$ for $3 \mathrm{~min}$, followed by 30 cycles of amplification $\left(94{ }^{\circ} \mathrm{C}\right.$ 30 second, $55^{\circ} \mathrm{C} 30 \mathrm{~s}$ and $72{ }^{\circ} \mathrm{C}$ for $\left.1 \mathrm{~min}\right)$. At the end, the reaction mixture was heated at $72{ }^{\circ} \mathrm{C}$ for $10 \mathrm{~min}$ and cooled down to $4^{\circ} \mathrm{C}$. After verification of correct amplification by electrophoresis on $1 \%$ agarose gel, the obtained PCR product was purified (GenUp ${ }^{\mathrm{TM}}$ PCR/Cleanup Kit, Biotechrabbit GmbH, Germany) and sequenced with the Sanger method. The final sequence was inserted into NucleotideBlast (PubMed), and the similarity with present ITS sequences was evaluated. Finally, the sequence was submitted into GenBank and is available under the Accession number: MK522147.

\section{Evaluation of T.atroviride 01 - plant interaction} The plant Lepidium sativum (Nohel Garden a.s., Dobříšs, Czech Republic) was used as a model plant. Sowing was preceded by seed disinfection. Seeds were submerged in Sanosil Super 25 Ag (S010) disinfection solution (Sanosil CZ s.r.o., Praha) and incubated for $10 \mathrm{~min}$. Then, Sanosil Super $25 \mathrm{Ag}$ was removed by pipetting, and this step was repeated 2-times. Then, seeds were swirled three times in sterile $\mathrm{H}_{2} \mathrm{O}$. As for the next stage, 10 seeds were placed on Murashige and Skoog nutrition medium in a glass Petri dish (diameter $20 \mathrm{~cm}$ ). The distance between seeds was approximately $1 \mathrm{~cm}$. For the first $24 \mathrm{~h}$, cultivation was performed in a horizontal position at laboratory temperature until seeds began to germinate. Then Petri dishes were placed at $60^{\circ}$ angle. $48 \mathrm{~h}$ after sowing, $5 \mathrm{~mm}$ disc of isolate mycelium was put in the position opposing forming roots. One week after inoculation, the morphology of plants was evaluated by measuring the length of the main root and counting the number of lateral roots.

\section{Determination of mycoparasitic activity by dual- culture assay}

Determination of mycoparasitic activity took place on PDA medium in Petri dish (diameter $10 \mathrm{~cm}$ ). First, $5 \mathrm{~mm}$ disc of the mycelium of phytopathogen was inoculated on the growth medium, close to the edge of Petri dish and after 4-day cultivation, at the laboratory temperature, T. atroviride $\mathrm{O} 1$ was inoculated at the place opposing pathogenic fungus and cultivation continued for next 13 days.

After this period, conidiation on the colony of $T$. atroviride $\mathrm{O} 1$, as well as on phytopathogen colony was determined. The number of conidia was evaluated by direct counting in the hemocytometer and by cultivation method. Briefly, the spore suspension was prepared by vortexing of two $5 \mathrm{~mm}$ disc of mycelia (cut out from the colony) in the $0.1 \%$
Tween 80 water solution (Biolife, Milan, Italy). The spore suspension obtained from the dual-culture zone (the zone of overgrowth) was prepared using the same procedure as mentioned previously, then it was diluted by factor 10 to $10^{-4}$, and $200 \mu \mathrm{L}$ of each dilution was spread on fresh PDA medium (Biolife, Milan, Italy). After short cultivation (48 h) colony forming units (CFU) were counted. Simultaneously, according to the morphology of obtained colony, we were able to evaluate if colonies of phytopathogenic fungi were present or not, meaning the fungistatic or fungicidal effect of $T$. atroviride O1 on plant pathogenic fungi.

\section{Production and isolation of SMs}

T. atroviride $\mathrm{O} 1$ was inoculated on PDA medium and cultivated in static circadian mode, at laboratory temperature for two weeks, so the colony entered stationary phase of growth when production of SMs is expected to culminate.

The isolation of metabolites was performed by using extraction into ethyl acetate (Centralchem, s.r.o., Bratislava, Slovakia). SMs were isolated from both mycelium and cultivation medium. Water present in the extract was removed using anhydrous $\mathrm{Na}_{2} \mathrm{SO}_{4}$ (Lachema Brno, Czech Republic) and the extract was concentrated by vacuum evaporation of ethyl acetate. Obtained metabolites were dissolved in a small amount of ethyl acetate. The tiny fraction was separated by Thin Layer Chromatography (TLC) in a mixture of benzene : acetone 3 : 1 (Centralchem, s.r.o., Bratislava, Slovakia) and then the solvent was removed entirely again. Concentrated SMs were dissolved in dimethyl sulfoxide (DMSO) (Centralchem, s.r.o., Bratislava, Slovakia).

\section{Antimicrobial activity of SMs}

For evaluation of antimicrobial activity, microdilution method was applied. Bacteria were cultivated in Mueller Hinton Broth (MHB), and yeasts were cultivated in Sabourad Glucose Broth (SGB) (Biolife, Milan, Italy). The overnight inoculum was prepared and used for inoculation of the growth media. Microorganisms were cultivated at $37^{\circ} \mathrm{C}$ under shaking $(350 \mathrm{rpm})$ in microtitration plates. To $198 \mu \mathrm{L}$ of $1 \%$ inoculum, $2 \mu \mathrm{L}$ of $\mathrm{SMs}$ dissolved in DMSO were added. Absorbance at $630 \mathrm{~nm}\left(\mathrm{~A}_{630}\right)$ was measured as the growth parameter. The measurement was performed until microorganisms reached the stationary phase of growth. The growth of microorganisms treated with an extract of isolated secondary metabolites was compared with control (inoculum with $1 \%$ DMSO) according to the formula (1) for the percentage of growth. 


$$
\%_{\text {growth }}=\frac{A_{630, \text { sample }}}{A_{630, \text { control }}} \times 100 \%
$$

The plate dilution method was used for the evaluation of the antifungal potential of secondary metabolites on plant pathogenic filamentous fungi. Three $\mathrm{mL}$ of PDA medium was supplemented with $30 \mu \mathrm{L}$ of the extract of SMs (in control, SMs were replaced by $30 \mu \mathrm{L}$ of DMSO, the solvent) and was poured in $3 \mathrm{~cm}$ diameter Petri dish. Once it solidified, it was inoculated with spore suspension in $0.1 \%$ Tween 80 water solution (A. alternata, $F$. culmorum) or with the mycelium disc of $B$. cinerea. The spore suspension $\left(5 \mu \mathrm{L}=10^{3}\right.$ conidia/disc) was applied on filter paper disc placed in the center of the growth media, or the growth medium was inoculated with a disc of the mycelium of $B$. cinerea (5 mm diameter). The filamentous fungi were cultivated at the laboratory temperature until the control reached its stationary phase of growth. The growth of fungi was evaluated by measuring the diameter of the fungal colony. The growth was compared to the growth of control $(3 \mathrm{~mL}$ of PDA medium with $30 \mu \mathrm{L}$ of DMSO). The evaluation was performed according to the following equation:

$$
\%_{\text {growth }}=\frac{\text { diameter of colony } y_{\text {sample }}}{\text { diameter of colony } y_{\text {control }}} \times 100 \%
$$

\section{MALDI-TOF analysis of produced peptaibiotics}

For isolation of peptaibiotics, the method of Neuhof et al. (2007) was used with some modifications. Isolate of Trichoderma spp. was cultivated on PDA medium covered with cellophane at laboratory temperature, in the circadian rhythm (sun-light), for 13 days to make sure that the production of SMs had already started. Peptaibiotics were extracted into acetonitrile/methanol/water (1: 1 : 1, by vol.) (Sigma Aldrich, St. Louis, USA), centrifuged (12000 rpm, $3 \mathrm{~min}$, microcentrifuge) and $1 \mu \mathrm{L}$ of the extract was mixed with $1 \mu \mathrm{L}$ matrix solution [10 mg of 2.5-dihydroxybenzoic acid $\mathrm{mL}^{-1}$ (Bruker Daltonics, Billerica, Massachusetts, USA) in acetonitrile/methanol/water ( $1: 1: 1$, by vol.) and $0.3 \%$ trifluoroacetic acid (Sigma Aldrich, St. Louis, USA)]. One $\mu \mathrm{L}$ of the sample - matrix mixture was directly spotted onto the target plate (MTP 384 plate ground steel BC, Bruker Daltonik GmbH, Germany) and allowed to air dry before analysis. Alternatively, mycelium was disintegrated in liquid nitrogen, and then peptaibiotics were extracted in $60 \%$ ethanol. After centrifugation (12000 rpm, $3 \mathrm{~min}$, microcentrifuge), $1 \mu \mathrm{L}$ of the extract was mixed with $1 \mu \mathrm{L}$ matrix solution, and $1 \mu \mathrm{L}$ of the resulting solution was spotted onto a MALDI target plate.
Measurements were performed in a reflector positive mode. Protein standard (Bruker Daltonics, Billerica, Massachusetts, USA) solution served for calibration with $\mathrm{m} / \mathrm{z}$ between 700-3500 (Tab. 1). From obtained mass spectra, the peaks with highest $\mathrm{m} / \mathrm{z}$ were identified, which represented the peptaibotics. Spectra of $\mathrm{m} / \mathrm{z}$ were compared with spectra described by Neuhof et al. (2007), as well as Comprehensive peptaibiotics database (Neuman et al., 2015), which helped to identify present peptaibiotics.

Tab. 1. Peptides and their $m / z$ (given by producer), which were in solution for calibration of mass spectrometer.

\begin{tabular}{lr}
\hline Protein & \multicolumn{1}{c}{$m / z$} \\
\hline Bradykinin (1-7) $[\mathrm{M}+\mathrm{H}]+$ mono & 757.399 \\
Angiotensin II $[\mathrm{M}+\mathrm{H}]+$ mono & 1046.542 \\
Angiotensin I $[\mathrm{M}+\mathrm{H}]+$ mono & 1296.685 \\
Substance P [M+H]+ mono & 1347.735 \\
Bombesin [M+H]+ mono & 1619.822 \\
Renin Substrate $[\mathrm{M}+\mathrm{H}]+$ mono & 1758.933 \\
ACTH-clip (1-17) $[\mathrm{M}+\mathrm{H}]+$ mono & 2093.086 \\
ACTH-clip (18-39) $[\mathrm{M}+\mathrm{H}]+$ mono & 2465.198 \\
Somatostatin $(28)[\mathrm{M}+\mathrm{H}]+$ mono & 3147.471 \\
\hline
\end{tabular}

\section{Results and discussion}

\section{Identification of obtained fungal isolate}

Biological material was isolated from the forest around the village Zázrivá, located in the northern part of Slovakia; region Orava. The underlying assumption in our work was the fact that Trichoderma sp. is a saprophytic filamentous fungus, which uses its cellulolytic enzymes for degradation of plant material. After isolation of pure culture (Fig. 1), we observed green concentric rings on fungal colonies with soft mycelium and coconut aroma - feature typical for Trichoderma sp. Secre-

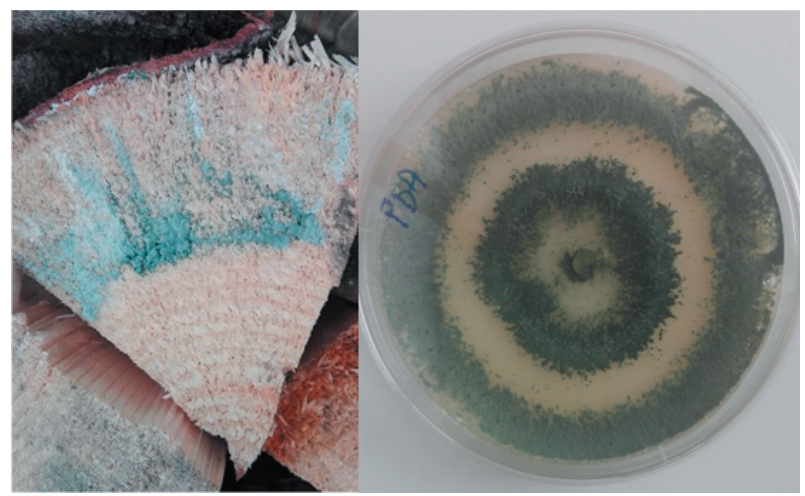

Fig. 1. Colony of isolate of Trichoderma sp. on natural substrate and Potato-Dextrose Agar. 


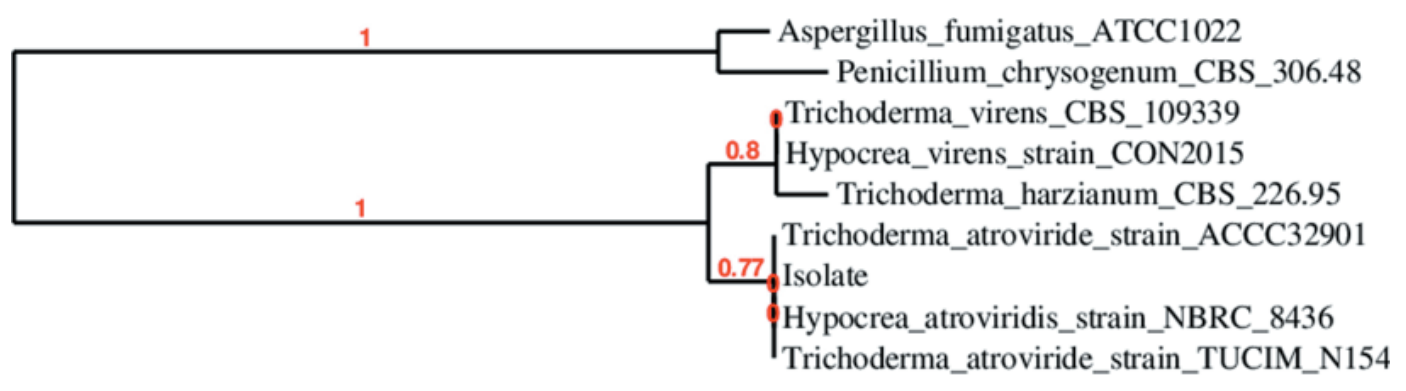

0.08

Fig. 2. Phylogenetic analysis of T. atroviride $\mathrm{O} 1$ isolate.

tion of yellow-green guttation droplet was also observed on the colony.

The phenotypic analysis was followed by genetic identification of ITS region, localized between ITS1 and ITS4 primers. After obtaining the genomic DNA, amplification of the desired region and its sequencing by Sanger method, the resulting sequence compared with GeneBank was similar to the ITS region of Trichoderma atroviride at $99 \%$. The sequence was submitted into GenBank database and is available under the Accession number MK522147. Identity was also confirmed by insertion into a phylogenetic tree (Dereeper et al., 2008 [http://www. phylogeny.fr]), and the classification of the isolate was noticeable (Fig. 2).

\section{Growth promotion of the model plant L. sativum}

Due to well-known abilities of Trichoderma sp. to promote plant growth, the characterization of isolate started in this field. After one week of cocultivation with $L$. sativum, the positive effect on the model plant was visible (Fig. 3). For both, control plants (not exposed to T. atroviride O1) and plants exposed to $T$. atroviride $\mathrm{O} 1$, the length of the primary root was similar $(11 \pm 0.8 \mathrm{~cm}$ and $11 \pm 0.9 \mathrm{~cm}$, respectively), while the number of lateral roots

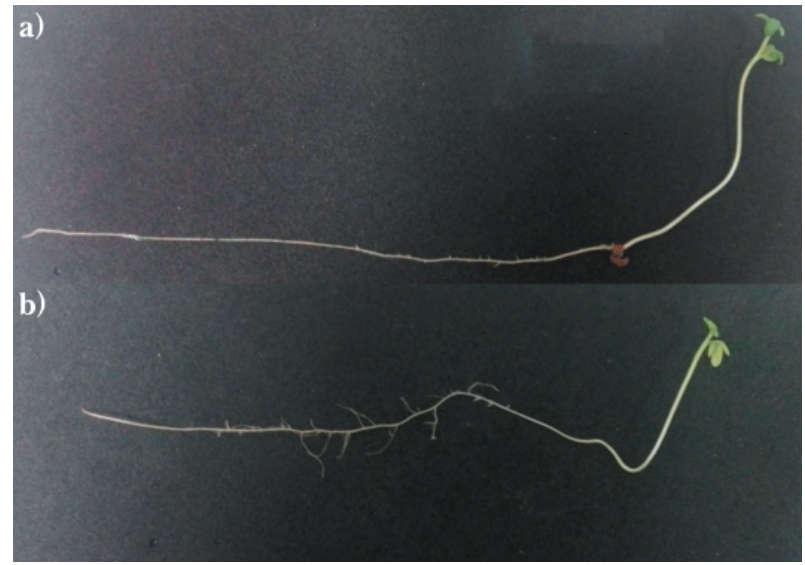

Fig. 3. The growth of L. sativum: a) control; b) inoculated plant with $T$. atroviride $\mathrm{O} 1$. showed a significant increase. For control plants, we detected only $5 \pm 1$ lateral roots per plant. The number of lateral roots of inoculated plants increased to $19 \pm 2$ per plant. Obviously (Fig. 3), so did the length of these roots (instead of a couple of millimeters, the longest lateral root was around $1 \mathrm{~cm}$ long). To conclude, it is highly probable that the isolate T. atroviride $\mathrm{Ol}$ promotes plant growth in the way that stimulates the development of lateral roots. The observed phenotype is very similar to the observed effect of auxins and auxin-like compounds - the elicitors of plant growth. Auxins and auxin-like compounds act as signal molecules for stimulation of genes involved in plant growth. The effect of lateral roots promotion was observed for indole-3-acetic acid (IAA), one of the most studied compounds in the area of biocontrol of the plant growth (Druzhinina et al., 2011). Recently, the focus on the role of SMs in this process is increased because of the discoveries of several metabolites with the same growth-promotion effect. Among SMs described as elicitors of plant growth, 6-PP, peptaibols, harzianolides, and polyketide chrysophanol are known (Liu et al., 2016, Zeilinger et al., 2016; Mukherjee et al., 2013; Vinale et al., 2008a).

\section{The mycoparasitic activity of T. atroviride 01}

The mycoparasitism is a trophic relationship, where a parasitic fungus lives in association with host fungus while derives the nutrients from it (Mukherjee et al., 2013). It is one of the main features of Trichoderma sp., which uses its enzymatic apparatus (cell wall degrading enzymes) to attack the colony of phytopathogen (host organism) (Druzhinina et al., 2011). Our experiment revealed that the isolate $T$. atroviride $\mathrm{O} 1$ is not only able to attack the colonies of three different phytopathogens, including F. culmorum but also wholly overgrows host fungi (Fig. 4). Fusarium sp. is one of the most problematic plant pathogens due to its production of trichothecenes (Tijerino et al., 2011). The mycoparasitic activity was evaluated by conidiation criteria and by the ability of the fungus 


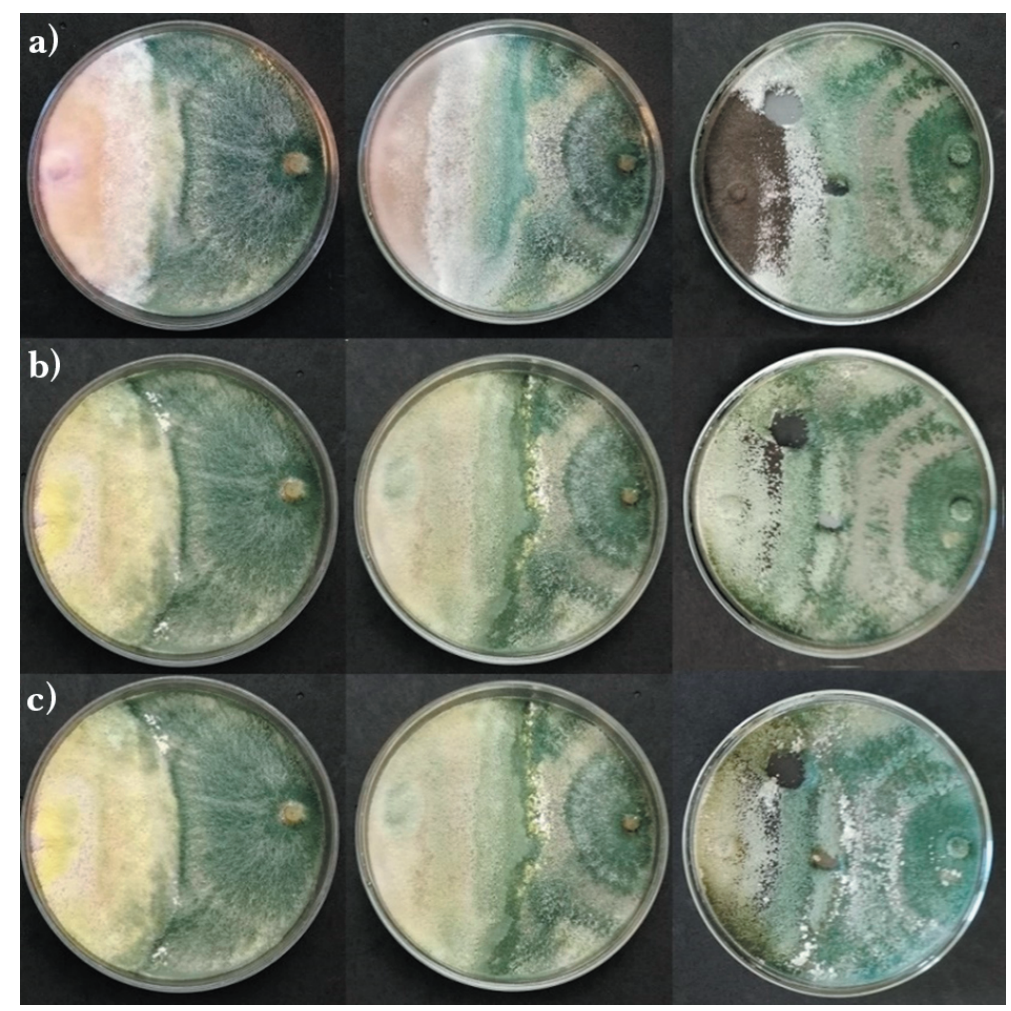

Fig. 4. Dual-culture of T. atroviride $\mathrm{O} 1$ and F. culmorum (on the left), B. cinerea (in the middle), and $A$. alternata (on the right) after a) 6-day; b) 10-day; c) 13-day cultivation.

to overgrow the colony of phytopathogen. We observed that the intensity of conidiation of T. atroviride $\mathrm{O} 1$ was decreased. At the zone of overgrowth, the conidiation reached $10^{5}-10^{6}$ conidia per $\mathrm{cm}^{2}$. The effect of isolate on all phytopathogens was fungicidal. Phytopathogens were not able to grow in fresh culture medium after the attack of $T$. atroviride $\mathrm{O} 1$. To sum up, it is clear that the isolate T. atroviride $\mathrm{O} 1$ showed high potential for biocontrol processes.

\section{Secondary metabolites with antimicrobial potential}

Production of secondary metabolites (SMs) of Trichoderma sp. peaks in the late stationary phase, when specific metabolites can be detected, for instance, 6-pentyl- $\alpha$-pyrone (6-PP), which is a volatile organic compound responsible for coconut aroma, typical of Trichodermasp. (Collins and Halim, 1972), with observed antifungal and plant-promoting activity (Vinale et al., 2008a). Such coconut aroma (production of 6-PP) was noticeable fairly soon, specifically after one week of cultivation, when the production of yellow-green guttation droplets was also observed, and the growth PDA medium was changing its colour in the way of getting a yellow tint. At this time, secondary metabolites were extracted in the ethyl acetate. Obtained concentrated extract of metabolites had a brownish color. The TLC analysis of the metabolic profile of T. atro- viride $\mathrm{O} 1$ is shown in Fig. 5. Together, eight spots were detected (over the limit of detection) what, presumably, represent eight different types of metabolites, according to their polarity. Metabolites (at the start line of chromatogram) are considered to be most polar compounds, unable to separate in benzene : acetone $3: 1$. Furthermore, these spots

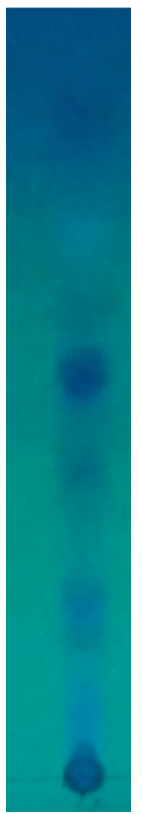

Fig. 5. TLC chromatogram of SMs of T. atroviride O1 detected under UV light $(254 \mathrm{~nm})$. 
were the only observable metabolites in visible light, in which they were brown.

The involvement of $\mathrm{SMs}$ in the interaction of Trichoderma sp. with organisms such as plants (growth promoting), phytopathogens (mycoparasitism), and other microorganisms have been extensively studied and reviewed (Zeilinger et al., 2016, Antasova et al., 2013, Malmierca et al., 2012, Druzhinina et al., 2011, Vinale et al., 2008a). In our work, we have focused on the inhibition effect of SMs of T. atroviride $\mathrm{O} 1$ on model bacteria, yeasts, and phytopathogenic filamentous fungi. Except extracted mixture, 2-times $(2 \times)$ and 4-times $(4 \times)$ diluted mixtures of obtained metabolites were used, in order to detect antimicrobial effect dependent on their concentrations.

Antibacterial activity of SMs is presented in Fig. 6 . It is clear that the growth of gram-negative bacteria E. coli was not influenced, while inhibition activity was intense in the case of gram-positive bacteria $S$. aureus and S. epidermidis. In the presence of SMs, the growth of S. aureus reached only $40 \%$, in comparison with control. Moreover, the extract of SM showed the $90 \%$ inhibition effect on the growth of
S. epidermidis. In both cases, the inhibitory effect was concentration dependent. Therefore, it may be suggested that $\mathrm{SM}$ of the isolate of $T$. atroviride O1 can play a role as antibacterial compounds.

SMs have shown antifungal activity on model yeasts (Fig. 7). In comparison with the control, only $7 \%$ growth of C. albicans was observed when the nondiluted extract was used, while the percentage of growth has increased to $30 \%$ and $32 \%$, when $2 \times$ and $4 \times$ diluted mixtures, respectively, were added. C. parapsilosis was also significantly affected. The addition of undiluted extract resulted in very similar growth inhibition to C. albicans; only $8 \%$ growth has been observed (Fig. 7). Further study is needed in order to identify the inhibition effect of either one metabolite or, more probable, to the mixture of metabolites to evaluate the possible synergistic activity of SMs.

When it comes to plant pathogenic fungi, the inhibition effect of SMs was not as strong as by yeast. $B$. cinerea was not inhibited at all, while the growth of other two fungi was at just under $75 \%$ in comparison with the control, namely at $74 \%$ for $A$. alternata and $73 \%$ for F. culmorum (Tab. 2). Based

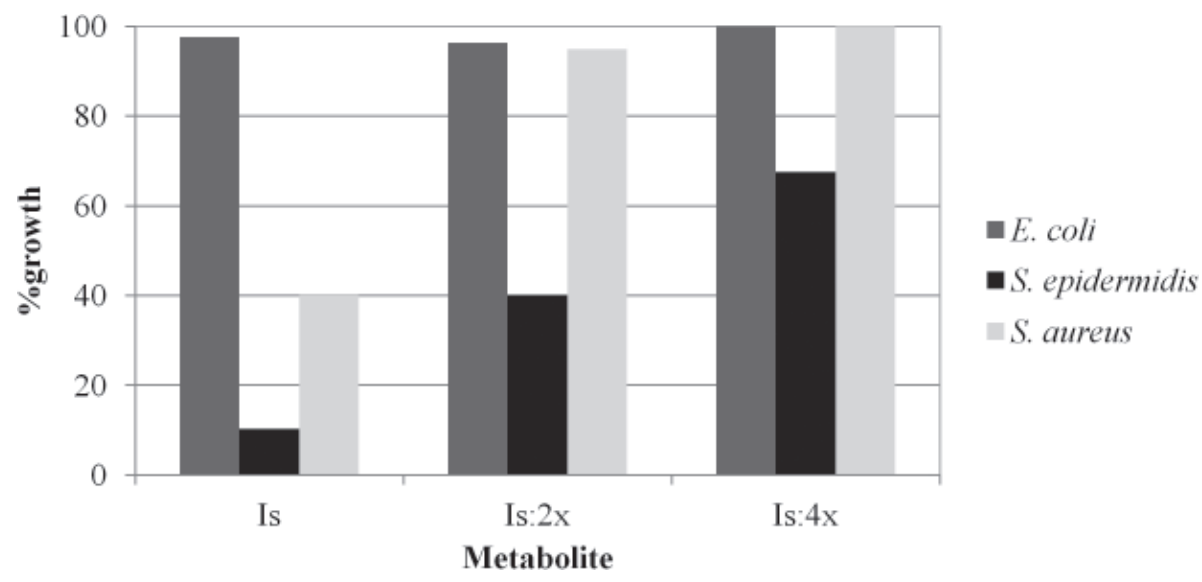

Fig. 6. Growth of model bacteria in the presence of SMs of T. atroviride O1. Is stands for non-diluted extract, Is: $2 \times-2$-times diluted, Is: $4 \times-4$-times diluted.

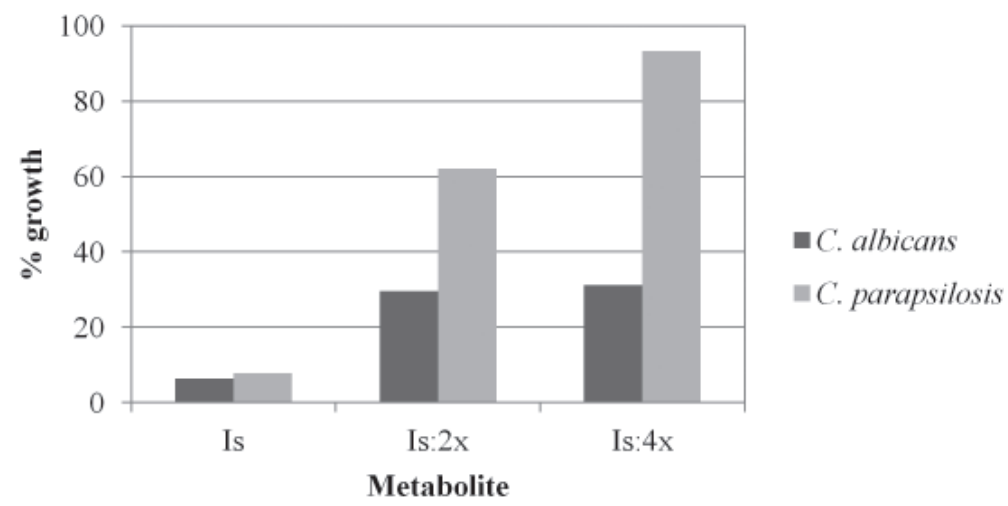

Fig. 7. Growth of C. albicans and C. parapsilosis in the presence of SM of T. atroviride O1. Is stands for non-diluted extract, Is: $2 \times-2$-times diluted, Is: $4 \times-4$-times diluted. 
on this result, it can be assumed that SMs plays a secondary role in the mycoparasitic attack of $T$. atroviride (the foremost importance still belongs to hydrolytic enzymes).

Tab. 2. Growth of phytopathogenic fungi in the presence of SMs of T. atroviride O1. Is stands for non-diluted extract, Is: 2x-2-times diluted, Is: 4x-4-times diluted.

\begin{tabular}{lcc}
\hline \multirow{2}{*}{ Metabolites } & \multicolumn{2}{c}{ \% growth } \\
\cline { 2 - 3 } & A. alternata & F. culmorum \\
\hline Is & 74 & 73 \\
Is: $2 \times$ & 87 & 82 \\
Is: $4 \times$ & 97 & 85 \\
\hline
\end{tabular}

\section{MALDI-TOF analysis of peptaibiotics produced by $T$. atroviride 01}

Peptaibiotics are non-ribosomal peptides with a length of 4-21 residues and a molecular weight of 500-2100 Da. Linear chain and presence of noncanonical amino acids ( $\alpha$-aminoisobutyric acid, isovaline) are typical for this group of SMs (Neumann et al., 2015). MALDI-TOF analysis of mass spectra allows us to determine the production of peptaibiotics (Neuhof et al., 2007). In this experiment, we also analyzed strain of Trichoderma viride F534 from Czech Collection of Microorganisms. According to the publication of Neuhof et al. (2007), samples for analysis were prepared by extraction into two different solvents. One of them was the mixture of acetonitrile : methanol : water (AMW) (1:1:1, by volume) and the alternative was $60 \%$ ethanol. We compared the mass spectra of these two extracts (Tab. 3) and found out that they are comparable. The similarities between strains depended on a range of molecular weight we were analyzing. Peaks m/z values ranging from 1900 to 2000 were almost the same (Fig. 8), according to the Comprehensive peptaibiotics database, they most probably correspond to the 20-21 amino acid long peptaibols (group of peptaibiotics), named alamethicins, paracelsins, suzukacillins and atroviridines (Tab. 4) - SMs, which production was described for Trichoderma sp. (Reino et al., 2008). Besides, alamethicins are known for their antifungal activity as well as signal functions in plant growth stimulation (Vinale et al., 2008b). On the other hand, the spectrum of lower $\mathrm{m} / \mathrm{z}$ was completely different (Fig. 9). Their identity was not revealed due to significant differences with values in the publication of Neuhof et al. (2007) and in the Comprehensive peptaibiotics database. However, we were able to estimate the length of peptides according to data in previously mentioned works. $\mathrm{m} / \mathrm{z}$ 1008 and 1024 are, presumably, 8-10 amino acids long, while $\mathrm{m} / \mathrm{z} 700-750$ should have $6-8$ residues, and m/z 600-700 4-6 amino acids. Peptides in these values can be either new peptaibiotics or fragments of longer peptaibiotics, as was suggested by Brito et al. (2014). This experiment was only a first step towards a potential study of peptaibiotics in isolate T. atroviride $\mathrm{O} 1$.

Tab. 4. Identities of some detected peptaibiotics.

\begin{tabular}{llc}
\hline$m / z$ & Name & $\begin{array}{c}\text { Number } \\
\text { of amino acids }\end{array}$ \\
\hline $\begin{array}{l}\text { 1949; 1963; } \\
1964 ; 1977 ; 1991\end{array}$ & Alamethicin F-30 & 20 \\
\hline $\begin{array}{l}\text { 1934; 1948; } \\
1962 ; 1976 ; 1990\end{array}$ & Alamethicin F-50 & 20 \\
\hline $1908 ; 1922 ;$ & Paracelsin A-I & 20 \\
1936 & Suzukacillin A 1-11 & 20 \\
\hline 1908,1922, & Atroviridin A-D & 20 \\
\hline 1936,1950 & Alamethicin type & 21 \\
\hline 1948,1962 & Alamethicin type & 21 \\
\hline 2016 & Paracelsine type & 21 \\
\hline 2001 & & \\
\hline 2002 & & \\
\hline
\end{tabular}

Tab. 3. Petaibiotics obtained from Trichoderma sp. after two different types of extraction procedure. Underlined are with intensity over $0.8 \times 10^{4}$ arbitrary units (a.u.).

\begin{tabular}{|c|c|c|}
\hline Trichoderma sp. & Method & Peaks of mass spectrum $[\mathrm{m} / \mathrm{z}]$ \\
\hline \multirow{2}{*}{ T. viride $\mathrm{F} 534$} & $\mathrm{EtOH}$ & $\begin{array}{l}681 ; 756 ; 769 ; 782 ; 804 ; 813 ; 820 ; 994 ; 1008 ; 1024 ; 1935 ; 1949 ; 1963 ; 1972 ; 1976 ; \\
1988 ; 2002 ; 2016\end{array}$ \\
\hline & AMW & $\begin{array}{l}621 ; 637 ; 665 ; 681 ; 709 ; 725 ; 756 ; 769 ; 782 ; 797 ; 813 ; 820 ; 857 ; 885 ; 901 ; 945 ; 989 \\
1008 ; \underline{1024} ; 1033 ; 1905 ; 1919 ; \underline{1935} ; \underline{1949} ; \underline{1963} ; \underline{1977} ; \underline{1988} ; \underline{2002} ; 2016\end{array}$ \\
\hline \multirow[t]{2}{*}{ T. atroviride $\mathrm{O} 1$} & $\mathrm{EtOH}$ & $\begin{array}{l}\underline{621} ; 637 ; \underline{665} ; 681 ; 699 ; \underline{709} ; 725 ; 740 ; \underline{753} ; 769 ; 797 ; 804 ; 813 ; 841 ; 857 ; 885 ; 901 ; \\
929 ; 945 ; 973 ; 989 ; 1008 ; 1017 ; 1024 ; 1033 ; 1061 ; 1077 ; 1121 ; 1149 ; 1193 ; 1919 ; 1933 ; \\
1947 ; 1956 ; 1963 ; 1972 ; 1986 ; 2002 ;(2010 ; 2016 ; 2024)\end{array}$ \\
\hline & AMW & $\begin{array}{l}621 ; 637 ; 665 ; 681 ; 709 ; 725 ; 758 ; \underline{782} ; \underline{804} ; \underline{820} ; 857 ; 901 ; 945 ; 992 ; \underline{1008} ; \underline{1024} ; \\
1033 ; 1077 ; 1340 ; 1905 ; \underline{1933} ; \underline{1919} ; \underline{1949} ; \underline{1963} ; \underline{1972} ; \underline{1988} ; \underline{2002} ; \underline{2016}\end{array}$ \\
\hline
\end{tabular}




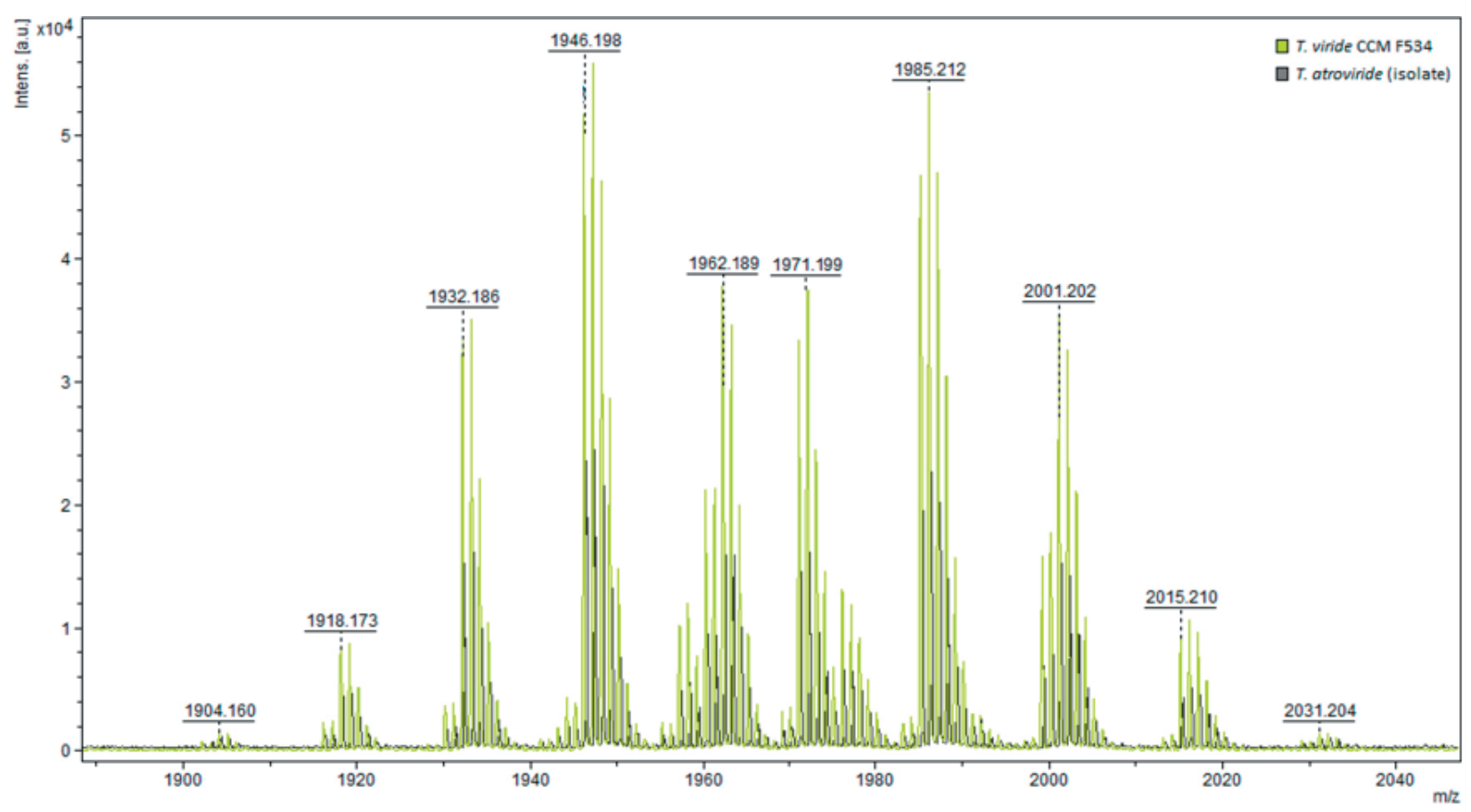

Fig. 8. Mass spectra of Trichoderma sp. between m/z values 1900-2000.

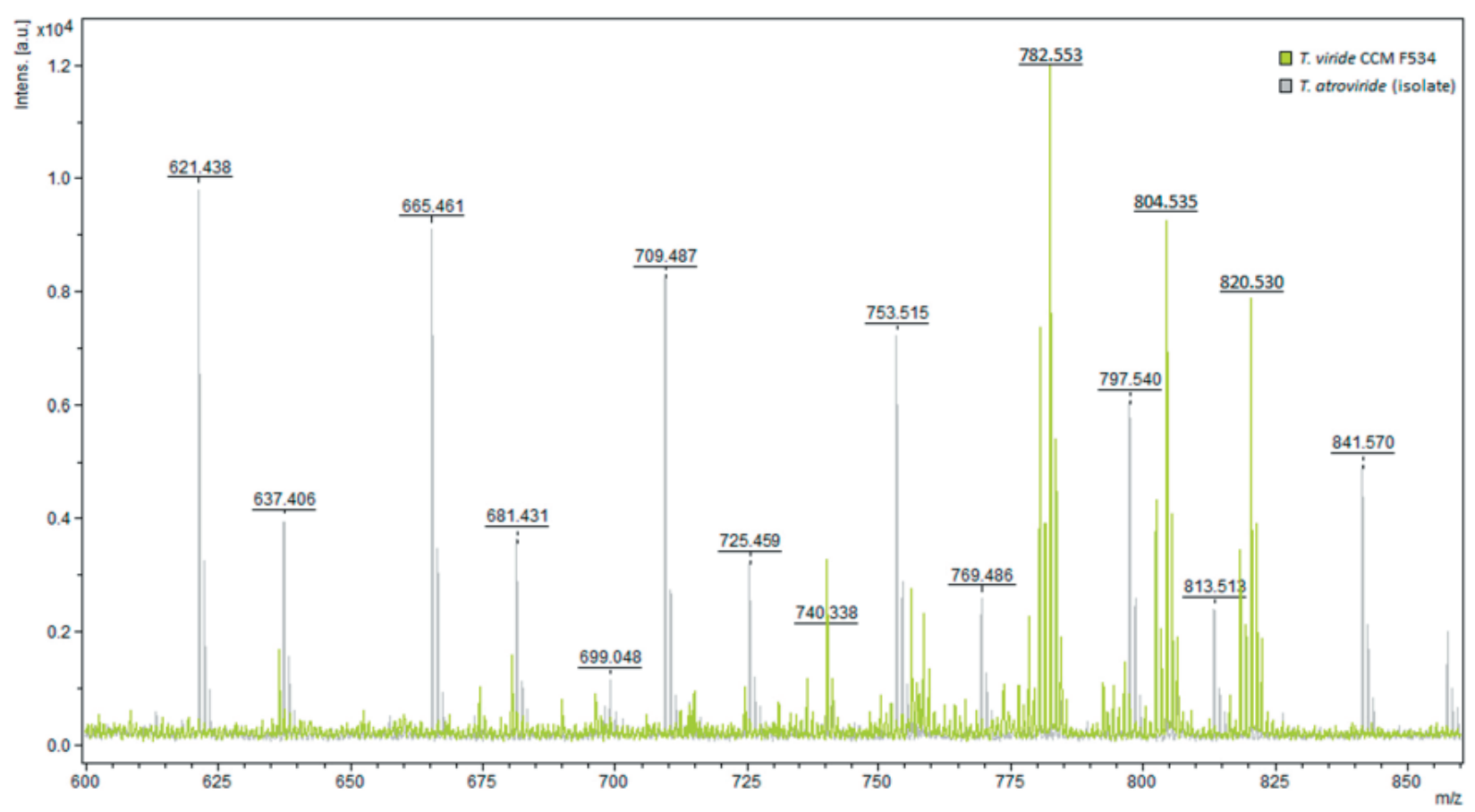

Fig. 9. Mass spectra of Trichoderma $\mathrm{sp}$. between $\mathrm{m} / \mathrm{z}$ values $600-825$.

\section{Conclusion}

The characterization of some essential features typical of Trichoderma sp. was conducted on isolate of $T$. atroviride $\mathrm{O} 1$ obtained from the environment. Isolate has shown strong mycoparasitic activity, as well as the ability to promote plant growth. T. atroviride O1 has produced typical peptaibiotics described for Trichoderma sp. However, unknown peptides were also detected. The role of secondary metabolites seems to be auxiliary in the mycoparasitic attack, while the inhibition activity against gram-positive bacteria and yeast indicates the potential of this isolate for further study as a biocontrol agent, and as a source of antimicrobial compounds.

\section{Acknowledgment}

This work has been supported by the Slovak Scientific Grant Agency (VEGA 1/0697/18) and ITMS: 26230120006. 


\section{References}

Alwhibi MS, Hashem A, Abs Allah EF et al. (2017) Journal of Integrative Agriculture 16: 1751-1757.

Antasova L, Crom SL, Gruber S et al. (2013) BMC Genomics 14: 121.

Antasova L, Knox BP, Kubicek CHP et al. (2013) Eukaryotic Cell 12: 1499-1508.

Brito JPC, Ramada MHS, de Magalhães MTQ, Silva LP, Ulhoa CJ (2014) Springerplus 3: 600.

Collins RP, Halim AF (1972) Journal of Agricultural and Food Chemistry 20: 437-438.

Dereeper A, Guignon V, Blanc G et al. (2008) Nucleic acid research 36: 465-469.

Druzhinina I, Seidl-Seiboth V, Herrera-Estrella A, Horwitz BA et al. (2011) Nature reviews. Microbiology 9: 749-759.

Harman GE, Howel CR, Viterbo A, Chet I, Matteo L (2004) Nature reviews. Microbiology 2: 43-56.

Liu SH, Liao CHK, Lo CHT et al. (2016) Physiological and Molecular Plant Pathology 96: 1-7.
Mukherjee PK, Horwitz BA, Herrera-Estrella A, Schmoll M, Kenerley CHM (2013) Annual Review of Phytopathology 51: 105-129.

Nawrocka J, Małolepsza U (2013) Biological Control 67: 149-156.

Neuhof T, Dieckmann R, Druzhinina I, Kubicek CP, von Döhren H (2007) Microbiology 153: 3417-3437.

Neumann NKN, Stoppacher N, Zeilinger S et al. (2015) Chemistry \& Biodiversity 12: 743-751.

Reino JL, Guerrero RF, Hernández-Galán R, Collado IG (2008) Phytochemical reviews 7: 89-123.

Tijerino A, Cardoza RE, Moraga J et al. (2011) Toxins 3: 1220-1232.

Vinale F, Sivasithamparam K, Ghisalberti EL et al. (2008a) Soil Biology \& Biochemistry 40: 1-10.

Vinale F, Sivasithamparam K, Ghisalberti EL et al., (2008b) Physiological and Molecular Plant Pathology 72: 80-86.

Zeilinger S, Gruber S, Bansal R, Mukherjee PK (2016) Fungal Biology Reviews 30: 74-90. 